\title{
Cirugia transeptoesfenoidal en adenomas hipofisarios productores de prolactina
}

\author{
O. López-Arbolay; O. Morales-Sabina; J.L. González-González y N. Valdés-Lorenzo
}

Servicio de Neurocirugía. Hospital Clínico Quirúrgico "Hermanos Ameijeiras". Ciudad de la Habana. Cuba.

\section{Resumen}

Introducción. Para un gran número de tumores pituitarios, la cirugía es el tratamiento de primera línea y el abordaje transesfenoidal microquirúrgico el proceder más utilizado. El tratamiento actual de los prolactinomas es bastante controversial, por los buenos resultados alcanzados con los nuevos agonistas dopaminérgicos; no obstante la adenomectomía selectiva se incluye entre las modalidades de tratamiento para aquellos prolactinomas que no respondan a dicho tratamiento dopaminérgico.

Objetivo. Evaluar los resultados del tratamiento microquirúrgico por vía transeptoesfenoidal de los adenomas productores de prolactina en el Servicio de Neurocirugía del Hospital "Hermanos Ameijeiras".

Material y método. Presentamos un estudio retrospectivo y descriptivo de 63 pacientes intervenidos por vía sublabial transeptoesfenoidal microquirúrgica, portadores de este tipo de adenomas, y tratados en nuestro servicio desde 1996 hasta el 2003. Se analizan edad, sexo, cuadro clínico, tamaño de las lesiones, niveles hormonales, complicaciones y evolución postoperatoria.

Resultados. Se encontró un franco predominio del sexo femenino, $86 \%$ (54 pacientes). Se operaron 31 pacientes con macroadenomas y 32 con microadenomas. La complicación más común en el postoperatorio fue la diabetes insípida transitoria (11 enfermos), siendo de poca trascendencia. Se logró reducir las cifras iniciales de prolactina a valores no tumorales en el $\mathbf{9 0 . 6 \%}$ de lo microadenomas ( 29 casos) y en el $67.7 \%$ de los macroadenomas (21 casos). Los síntomas más frecuentes que aquejaban a nuestros pacientes mejoraron, sobre todo la cefalea en el $82 \%$ ( 36 casos) y los trastornos visuales en el $69 \%$ (18 casos).

Conclusiones. Se concluye que la adenomectomía transeptoesfenoidal es un proceder seguro y eficaz como opción de tratamiento para pacientes con adenomas secretores de prolactina que tengan indicación quirúrgica.

Recibido: 8-04-05. Aceptado: 19-05-05
Transsphenoidal approach to prolactinomas

Summary

Introduction. Transeptal transsphenoidal surgery for pituitary tumors is a well established surgical technique. In particular the use of medical treatment in patient with prolactinomas has induced the control of hiperprolactinemia and the shrinkage of the tumor in the great majority of the patients, for that reason the treatment of the prolactinomas is controversial.

Objective. We evaluate the results of trassphenoidal microsurgical treatment of prolactin secreting adenomas at our Unit.

Methods. We made a retrospective analysis of 63 patients operated on via transsphenoidal microsurgical technique for prolactin secreting adenomas between 1996 and 2003. Age, sex, symptoms, tumor size, hormonal levels, complications and postsurgical outcome werw considered.

Results. There was a female predominance of $86 \%$ of the cases and middle aged patients were more commonly seen 31 patients had tumors more than $10 \mathrm{~mm}$ in diameter on the CT scan. The most frecuent complication was transient diabetes insipidus (11 cases). Prolactin levels were reduced to non tumoral values in $90.6 \%$ of microadenomas ( 29 cases) and in $67.7 \%$ of macroadenomas (21 cases). Headache was the most frecuent complain in our patients with amelioration after surgery in $82 \%$ (36 cases). Campimetric visual defects werw reduced in $69 \%$ (18 cases).

Conclusions. Transsphenoidal adenomectomy is a safe treatment option for patients with prolactin secreting adenomas with surgical indication.

Introducción

Los tumores hipofisarios constituyen entre un $10 \mathrm{y}$ un $15 \%$ de las neoplasias intracraneales ${ }^{8,9,11,22}$. Estos tumores generalmente son adenomas productores o no de algún tipo de hormona ${ }^{1,9,11,17,22,24}$. 
Dentro de los adenomas se considera que los productores de prolactina (PRL), también conocidos como prolactinomas, constituyen aproximadamente de un 25 a un $65 \%$ de estas lesiones ${ }^{8,22}$.

Muchos tratamientos se han utilizado a lo largo de la historia. Se han empleado múltiples agonistas de la dopamina tratando siempre de minimizar sus efectos secundarios $^{4,10,23}$; diferentes modalidades de terapia radiante que van desde la clásica cobaltoterapia externa, hasta la radiocirugía estereotáxica en cualquiera de sus variantes ${ }^{14,19}$ y el abordaje quirúrgico que ha evolucionado desde finales del siglo $\mathrm{X} 1 \mathrm{X}^{3,9,12,16}$. Así, la elección del tratamiento debe individualizarse y muchas veces se utiliza la terapia combinada.

En sus inicios, el abordaje quirúrgico fue exclusivamente intracraneal. Posteriormente se comenzaron a utilizar las vías extracraneales, pero con poco éxito por la escasa visibilidad y la alta tasa de complicaciones que presentaban. A pesar de ello, tuvo sus defensores y mucho más después del advenimiento de la era antibiótica, la introducción del fluoroscopio en el acto operatorio por Guiot y del microscopio quirúrgico por Hardy $y^{9,12,16}$.

En la actualidad, los abordajes transesfenoidales tienen gran aceptación para tratar lesiones selares ${ }^{6}$. Se buscan nuevos aditamentos para mejorar la visión de un área tan restringida y compleja y se ha introducido el apoyo endoscópico, el cual, en ocasiones, ha logrado reemplazar incluso al microscopio ${ }^{18}$ En nuestros días se utilizan indistintamente ambas vías teniendo indicaciones precisas cada una de ellas, $72,16,25$.

En Cuba la historia de los abordajes extracraneales se remonta a la década de los sesenta cuando el Dr. Díaz emplea una vía transantroesfenoidal a la región selar. Posteriormente el Dr. Junco utiliza la vía transeptoesfenoidal (TSE), la cual es introducida en nuestro Servicio en $1983^{12}$.

El tratamiento actual de los prolactinomas es bastante controversial y en la decisión del mismo influye significativamente el tamaño de la lesión. Para algunos autores está indicado comenzar por los agonistas de la dopamina con lo cual se logra reducción de la hiperprolactinemia, del tamaño tumoral y en ocasiones hasta la desaparición del tumor ${ }^{2,4}$.

Otros autores, sin embargo, prefieren operar por vía transesfenoidal con vistas a extirpar radicalmente el adenoma señalando la dificultad quirúrgica adicional (fibrosis) que entraña el uso previo de la bromocriptina (agonista dopaminérgico más utilizado en nuestro país) ${ }^{21}$. Solamente existe consenso general en plantear la poca utilidad de la radioterapia para la curación de estos pacientes como único método terapéutico ${ }^{19}$.

Debido a la frecuencia del prolactinoma y a los trastornos endocrinos que provoca nos decidimos a realizar un estudio retrospectivo de los pacientes operados por esta causa en el Servicio de Neurocirugía de nuestro Hospital entre enero de 1996 y diciembre del 2003.

\section{Pacientes y método}

Se realizó un estudio descriptivo, retrospectivo y lineal, de 63 pacientes con adenomas hipofisarios productores de prolactina intervenidos mediante abordaje transeptoesfenoidal en el Servicio de Neurocirugía del Hospital Clínico Quirúrgico "Hermanos Ameijeiras" desde Enero de 1996 a Diciembre del 2003. En todos los casos se realizó estudio hormonal, neuro-oftalmológico e imagenológico completo.

Se consideró como portador de adenoma secretor de prolactina a todo paciente que cumpliera los siguientes criterios:

1. Manifestaciones clínicas compatibles con adenoma secretor de prolactina.

2. Prolactina igual o mayor de $100 \mathrm{ng} / \mathrm{ml}(3200 \mathrm{mu} / 1)$

3. Tomografía Axial Computarizada (TAC) con contraste endovenos, con imagen compatible con adenoma hipofisario.

4. Inmunohistoquímica compatible con adenoma hipofisario productor de prolactina.

Mediante TAC se clasificaron las lesiones en microadenomas cuando medían menos de $10 \mathrm{~mm}$ y en macroadenomas si eran mayores de $10 \mathrm{~mm}$.

Se consideraron prolactinomas con criterio quirúrgico aquéllos que cumplieran los siguientes criterios.

\section{Criterios de inclusión:}

1. Microadenomas productores de prolactina con fallo al tratamiento médico o intolerancia a los agonistas dopaminérgicos.

2. Macroadenomas productores de prolactina.

Se empleó la técnica microquirúrgica sublabial transeptal transesfenoidal que proporcionó un acceso rápido y seguro a la silla turca.

Se consideró como mejoría de la hiperprolactinemia cuando ésta disminuyó a valores no tumorales (menos de $100 \mathrm{ng} / \mathrm{ml})$.

Se consideró mejoría de los síntomas y signos cuando los pacientes referían menos intensidad de ellos o cuando se comprobó al examen físico una disminución de los mismos en las consultas al mes, 3 meses y 6 meses de operados.

Los datos se obtuvieron de los expedientes clínicos mediante encuesta confeccionada al efecto. Se emplearon tablas de dos entradas para evaluar asociación entre variables. Los datos se analizaron utilizando el paquete estadístico SPSS/PC. 


\begin{tabular}{lcc}
\hline & $\begin{array}{c}\text { Tabla 1 } \\
\text { Grupos etáreos }\end{array}$ \\
\hline Edad & Número de pacientes & $\%$ \\
\hline Hasta 20 años & 3 & 5 \\
$20-29$ años & 23 & 36 \\
$30-39$ años & 9 & 32 \\
$40-49$ años & 6 & 14 \\
$50-59$ años & 2 & 10 \\
60 ó más años & 63 & 3 \\
Total & 2 & 100 \\
\hline
\end{tabular}

Tabla 2

Manifestaciones clínicas de la enfermedad

\begin{tabular}{lcc}
\hline Manifestaciones clínicas & $\mathrm{N}^{\mathrm{o}}$ pacientes & $\%$ \\
\hline Cefalea & 44 & 70 \\
Trastornos menstruales & 41 & 65 \\
Galactorrea & 27 & 43 \\
Trastornos visuales campimétricos & 26 & 41 \\
Disfunción sexual eréctil & 2 & 3 \\
Infertilidad con menstruaciones normales & 4 & 6 \\
Hipopituitarismo & 1 & 2 \\
\hline
\end{tabular}

\section{Resultados}

Se operaron 63 pacientes portadores de adenomas secretores de prolactina, por vía transeptoesfenoidal. El rango de edad varió entre 17 y 62 años y el $82 \%$ de los pacientes se encontraban en la tercera, cuarta y quinta décadas de la vida, con predominio entre los 20 y 29 años de edad (36\%) (Tabla 1). En cuanto al sexo predominó el femenino con una relación de 6:1. (Figura 1).

Los síntomas fundamentales que motivaron la asistencia al facultativo fueron la asociación de amenorrea - galactorrea en 22 pacientes (35\%), la cefalea en 18 pacientes $(29 \%)$ y con menor frecuencia las alteraciones visuales en 7 casos, la galactorrea aislada y los trastornos menstruales aislados ( 5 casos cada uno) y la disfunción sexual eréctil en dos hombres de los nueve que participaron en el estudio.

Cuatro mujeres con menstruaciones normales fueron remitidas de la consulta de infertilidad y esta fue la principal causa de la consulta (Tabla 2).

Al analizar el cuadro clínico completo que presentaba cada paciente encontramos que la cefalea fue el síntoma que se presentó con mayor frecuencia (70\%), seguida por los trastornos menstruales de cualquier tipo (65\%), la

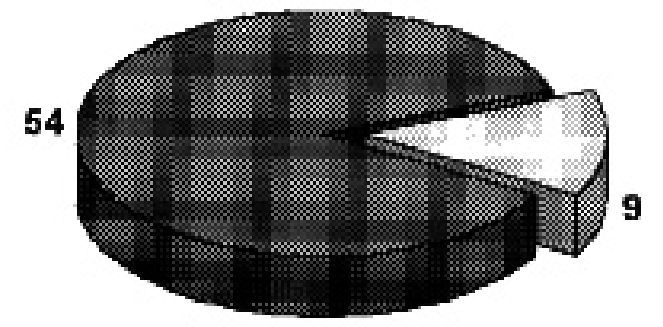
娄 Fem (86\%)
Masc(14\%)

Figura 1. Sexo.

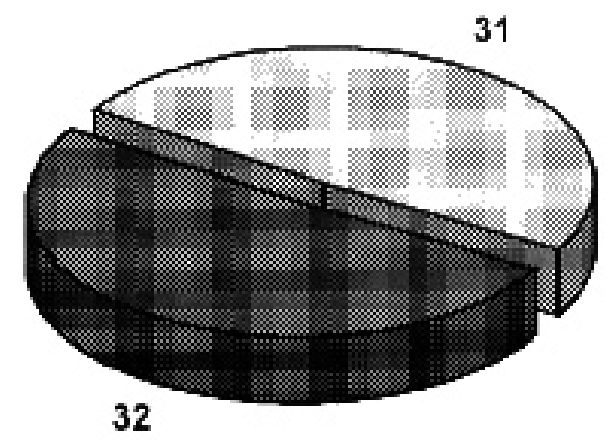
Micro(51\%)
BMacro( $49 \%)$

Figura 2. Clasificación imagenológica (TAC).

galactorrea (43\%) y los trastornos visuales campimétricos del tipo de hemianopsia heterónima bitemporal de grado variable (26\%). La disfunción sexual eréctil se presentó en sólo dos casos y la infertilidad con menstruaciones normales en 4. (Tabla 2).

Se operaron 32 microadenomas (51\%) y 31 macroadenomas (49\%). (Figura 2).

Las complicaciones postquirúrgicas más frecuente fueron la diabetes insípida transitoria que se presentó en 11 casos (17\%), tres pacientes presentaron fístula de LCR que resolvieron con tratamiento conservador. Dos pacientes tuvieron sinequias de la mucosa nasal y en un paciente se presentó epístaxis postoperatoria. (Figura 3).

Al mes de la operación las cifras iniciales de prolactina se redujeron a valores no tumorales en el $90.6 \%$ de 10 microadenomas (29 casos) y en el $67.7 \%$ de los macroadenomas (21 casos). (Figuras 4 y 5).

En las consultas de seguimiento se constató que la cefalea había mejorado en el $82 \%$ de los pacientes que la presentaban, los trastornos visuales campimétricos en el $69 \%$, la galactorrea en el $63 \%$ y los trastornos menstruales en el $54 \%$.(Tabla 3). 


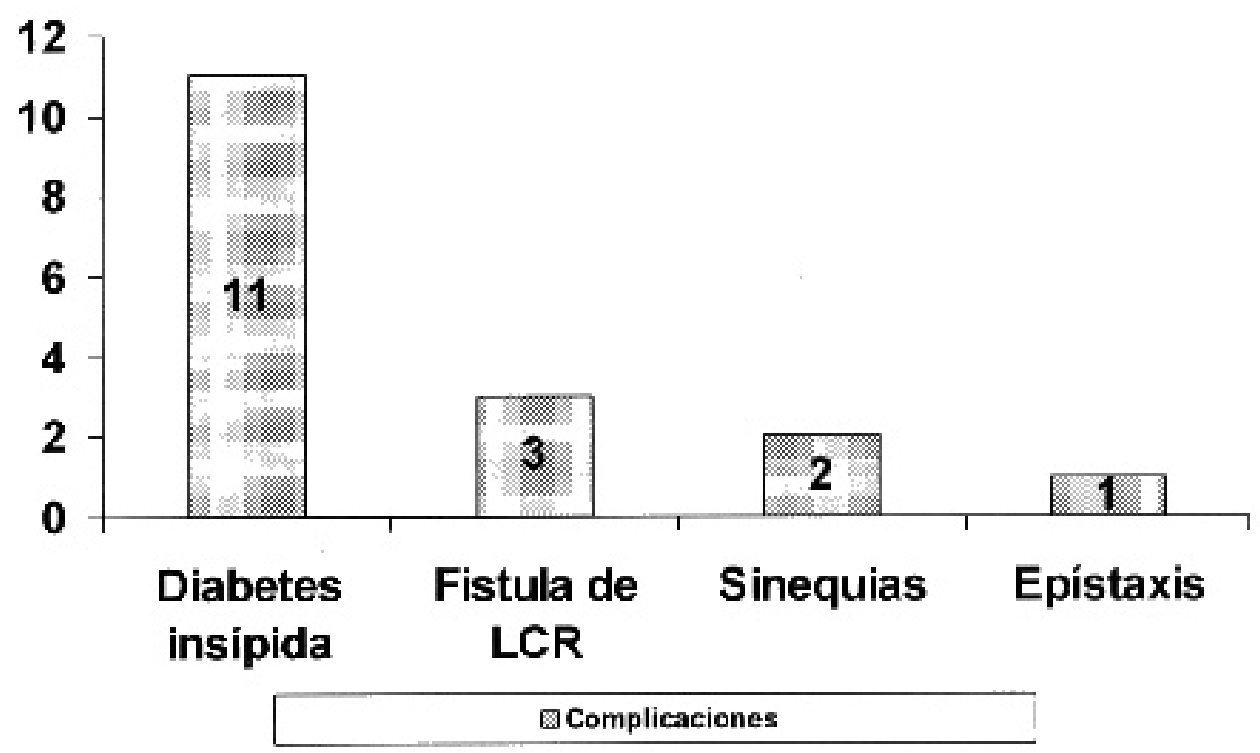

Figura 3. Complicaciones quirúrgicas
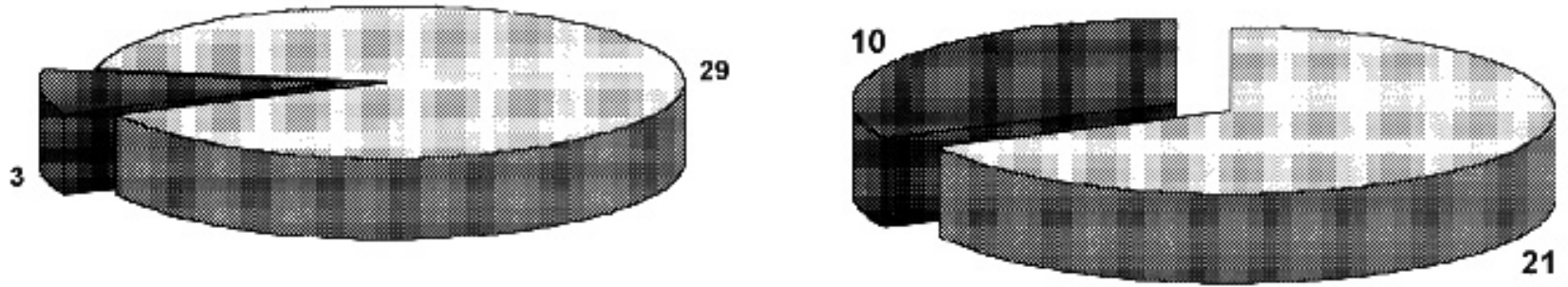

Dismunucion $\mathrm{m}$. No disminucion

UReduccion No reduccion

Figura 4. Reducción de los valores de prolactina a cifras no tumorales en microadenomas.
Figura 5. Disminución de los niveles de prolactina a cifras no tumorales en macroadenomas

Tabla 3

Evolución de los principales síntomas de la enfermedad

\begin{tabular}{lccccc}
\hline Síntomas & Total & Mejor & Igual & Peor & \% de Mejoría \\
\hline Cefalea & 4 & 36 & 7 & 1 & 82 \\
Trastornos visuales & 26 & 18 & 7 & 1 & 69 \\
Galactorrea & 27 & 17 & 10 & 0 & 63 \\
Trastornos menstruales & 41 & 22 & 19 & 0 & 54 \\
\hline
\end{tabular}

\section{Discusión}

Tal y como lo reportan la mayoría de los autores, en nuestra serie predominaron los pacientes del sexo femenino en edad férti1 $11^{17,12,15,16}$.

Generalmente son la galactorrea asociada a trastornos menstruales en las mujeres y las disfunciones sexuales en los hombres las causas más frecuentes que motivan la consulta de nuestros pacientes. Lo mismo observaron Often y cols. ${ }^{15}$ que plantean que la amenorrea en mujeres y la impotencia en hombres son los motivos de consulta más comunes. Beresin ${ }^{2}$ encontró que el $85 \%$ de 53 hombres con prolactinoma ingresaron con disminución de la función sexual.

En nuestro medio, la TAC ha resultado ser el estudio más utilizado para el diagnóstico del tumor. La relación 
entre micro y macroadenomas es variable según las series revisadas. Para la mayoría predominan las lesiones menores de $10 \mathrm{~mm}^{7,8,12,14}$ debido a que la disfunción endocrina se manifiesta primero que la clínica por aumento del volumen tumoral. Para otros sucede lo contrario y consideran más frecuentes los macroadenomas, sobre todo en hombres ${ }^{2,20}$.

Somos del criterio que los microadenomas son mucho más frecuentes que los macroadenomas, pero en nuestro estudio hemos tenido una cantidad similar de cada uno, simplemente porque son pocos los microadenomas que llegan a tener criterio quirúrgico por ser tratados con éxito, mediante agonistas de la dopamina (a diferencia de los macroadenomas). Nuestro trabajo no es un estudio epidemiológico de todos los casos con adenomas secretores de prolactina sino que solo incluye pacientes operados, donde los microadenomas incluidos han sido por fallo o intolerancia del tratamiento medicamentoso.

Se han reportado numerosas complicaciones tras la cirugía transeptoesfenoidal. En el postoperatorio, la diabetes insípida fue la complicación más frecuente, generalmente transitoria.

Algunas publicaciones se refieren a la recurrencia tumoral, hecho que todavía no hemos evaluado. A tal efecto algunos recomiendan el tratamiento médico como primera opción pues se obtienen similares resultados sin complicaciones propias del acto quirúrgico, aún en macroadeno$\operatorname{mas}^{2,4,5,13,15,18,20 \text {. }}$

En cuanto a la tasa de mortalidad quirúrgica en la actualidad se reporta que la misma es muy baja. En muchas ocasiones tiene relación con la experiencia previa del cirujano. Muchos, entre ellos nosotros, no hemos tenido ningún fallecido ${ }^{2,5,13}$. Giovanelli ${ }^{7}$ tuvo en 1000 pacientes operados un $1.6 \%$ de fallecidos entre 1970 y 1980 , y sólo un $0.5 \%$ entre 1981 y 1994.

La mejoría de la hiperprolactinemia es variable después del tratamiento quirúrgico. Para algunos la reducción hormonal se logra mejor con los agonistas de la dopamina ${ }^{2,4}$. Nosotros también utilizamos el tratamiento médico como primera opción y dejamos siempre el tratamiento quirúrgico para aquellos pacientes con macroadenomas (a los cuales le indicamos tratamiento médico unas semanas antes de la cirugía) y para microadenomas sin respuesta o intolerancia al tratamiento médico. Soule ${ }^{20}$ reportó un $45.5 \%$ de normoprolactinemia postoperatoria mientras que Feigenbaum ${ }^{5}$ encontró recurrencia de la hiperprolactinemia en el $47 \%$ de sus casos. No obstante, para Massoud ${ }^{13}$ el $90 \%$ (58 pacientes) tenían una prolactina normal después de la cirugía. En nuestra serie los valores de prolactina bajaron a valores no tumorales en el $90.6 \%$ de los microadenomas ( 29 casos) y en el 67.7\% de los macroadenomas (21 casos), aunque no siempre descendieron a cifras completamente normales.

El índice de curación de los pacientes también tiene sus variaciones. Varias series estudiadas reportan entre un 46 y un $87 \%$ siendo siempre mejor en casos con microadenomas $^{13,15,18}$.

Son conocidas las ventajas y las indicaciones del tratamiento médico para estos adenomas hipofisiarios pero parece ser que una vez que éstos tengan indicación quirúrgica lo más racional hoy en día es la combinación de la cirugía con otras modalidades terapéuticas (tratamiento médico y/o con radioterapia), previa valoración individual de cada paciente 6 .

Las pocas complicaciones y los resultados mostrados en el presente estudio, avalan el abordaje transeptoesfenoidal en nuestro medio, como un método de tratamiento útil y confiable para los adenomas productores de prolactina con criterio quirúrgico.

\section{Bibliografía}

1.Beauchesne, P., Trouillas, J., Barcal, F., Brunon, J.: Gonadotropic pituitary carcinoma. Case report. Neurosurgery 1995; 37: 810-816.

2. Beresin, M., Shimon, I., Madani, M.: Prolactinoma in 53 men: clinical characteristics and modes of treatment (male prolactinomas). J Endocrinol Invest 1995; 18: 436-441.

3. Cawley, C., Tindall, G.: Transsphenoidal surgery: operative techniques. En: Krisht A, Tindall A, eds. Pituitary disorders: comprehensive management. Baltimore: Lippincott, Williams and Wilkins, 1999: pp. 349-359.

4. Chanson, P.: Medical treatment of pituitary adenoma. Rev Prat 1996; 46: 1509-1513.

5. Feigenbaum, S.L., Downey, D.E., Wilson, C.B., Jaffe, R.B.: Transsphenoidal pituitary resection for preoperative diagnosis of prolactin secreting pituitary adenoma in women: Long term follow-up. J Clin Endocrinol Metab 1996; 81:17111719.

6. Greenberg, M.S.: Tumor. Handbook of Neurosurgery. New York; Greenberg Graphics Inc, 2001; pp. 386-495.

7. Giovanelli, M., Losa, M., Mortini, P.: Surgical therapy of pituitary adenomas. Metabolism 1996; 45 (8 Suppl 1): 115116.

8. Hung, S.: Estudios en pacientes con tumores hipofisarios y silla turca vacía primaria. Instituto Superior de Ciencias Médicas. Ciudad de la Habana. 1993. Tesis Doctoral.

9. Jane, J.A., Laws, E.R.: The surgical management of pituitary adenomas in a series of 3093 cases. J Am Coll Surg 2001; 193: 651-659.

10. Kamel, N., Vysal, A.R., Cesur, V., Erdogan, G., Baskal, N.: Normal growth and pubertal development during bromocriptine therapy in two patients with prolactinoma. J Endocr 1995; 42: 581-586.

11. Lubke, D., Saeger, W.: Carcinomas of the pituitary: Definition and Review of the literature. Gen Diagn Pathol 1995 ; 14: 81-92 
12. Maiquez, A.: Cirugía transeptoesfenoidal para afecciones de la región selar. Instituto Superior de Ciencias Médicas. Ciudad de la Habana. 1995. Tesis.

13. Massoud, F., Serri, O., Hardy, J., Somma, M., Beauregard, H.: Transsphenoidal adenomectomy for microprolactinoma: 10 to 20 years of follow-up. Surg Neurol 1996; 450: 341-346.

14. Motti, E.D., Losa, M., Pieralli, S., et al.: Stereotactic radiosurgery of pituitary adenomas. Metabolism 1996; 45(8 Suppl 1): 111-114.

15. Often, P., Rilliet, B., Reverdin, A., Demierre, B., Berney, J.: Pituitary adenoma secreting prolactin. Results of their surgical treatment. Neurochirurgie 1996; 42: 44-52.

16. Pardal, E.:Técnicas Quirúrgicas. En: Cirugía de la hipófisis y la región selar. $1^{\circ}$ ed. Buenos Aires : López Libreros Editores, 1984; pp. 161-79.

17. Saeger, W., Bosse, V., Pfingst, E., et al.: Prolacting producing hypophyseal carcinoma. Case report of an extremely rare metastatic tumor. Pathologe 1995; 16: 354-358.

18. Sethi, D.S., Pillay, P.K.: Endoscopic management of lessions of the sella turcica. J Laryngol Otol 1995; 109: 956962.

19. Sheline, G.E., Wara, V.M.: Radiation therapy of pituitary tumors. En: Youmans JR. Neurological Surgery. $3^{\circ}$ ed. Philadelphia; WB Saunders, 1990; pp. 3499-3503.

20. Soule, S.G., Farhi, J., Conway, G.S., Jacobs, H.S., Powell, M.: The outcome of hypophysectomy for prolactino- mas in the era of dopamine agonist therapy. Clin Endocrinol Oxf 1996; 440: 711-716.

21. Tindall, G.T., Barrow, D.L.: Tumors of the sellar and parasellar area in adults. En: Youmans JR. Neurological Surgery. $3^{\circ}$ ed. Philadelphia : WB Saunders, 1990; pp. 34473493.

22. Tomita, T., Gates, E.: Pituitary adenomas and granular cell tumors: incidence, cell type, and location of tumor in 100 pituitary glands at autopsy. Am J Clin Pathol 1999; 111 :817825.

23. Vázquez, J.E., Vázquez, E.: Tratamiento de los estados hiperprolactinémicos con lisurida en un estudio simple abierto. Ginec Obstet Méx 1991; 59: 202-205.

24. Wang, C.J., Howng, S.L.: Surgical management of TSH-secreting pituitary adenomas. Kao Hsiung I Hsueh Ko Hsueh Tsa Chih 1996; 12: 590-594.

25. Yasargil. M.G.: Transcranial surgery for large pituitary adenomas. En: Microneurosurgery of CNS Tumors. New York: Thieme Medical Publisher Inc, 1996; pp. 200-4.

López-Arbolay, O.; Morales-Sabina, O.; González-González, J.L.; Valdés-Lorenzo, N.: Cirugía transeptoesfenoidal en adenomas hipofisarios productores de prolactina. Neurocirugía 2006; 17: 226-231.

Correspondencia postal: San Lázaro 701. 10300 Centro Habana. Ciudad de la Habana. Cuba. 